\title{
A STUDY OF GRAPH CLOSED SUBSEMIGROUPS OF A FULL TRANSFORMATION SEMIGROUP
}

\author{
BY
}

\author{
R. G. BIGGS, S. A. RANKIN AND C. M. REIS
}

\begin{abstract}
Let $T_{X}$ be the full transformation semigroup on the set $X$ and let $S$ be a subsemigroup of $T_{X}$. We may associate with $S$ a digraph $g(S)$ with $X$ as set of vertices as follows: $t \rightarrow j \in g(S)$ iff there exists $\alpha \in S$ such that $\alpha(i)=j$. Conversely, for a digraph $G$ having certain properties we may assign a semigroup structure, $S(G)$, to the underlying set of $G$. We are thus able to establish a "Galois correspondence" between the subsemigroups of $T_{X}$ and a particular class of digraphs on $X$. In general, $S$ is a proper subsemigroup of $S \cdot g(S)$.
\end{abstract}

1. Introduction. In this article, we shall focus our attention on graph closed subsemigroups of $T_{X}$, i.e., subsemigroups $S$ for which $S \cdot g(S)=S$. As might be expected, there is a close relationship between this class of semigroups and the "geometry" of the associated graphs.

The class of graph closed nil subsemigroups is particularly amenable to treatment via the "geometric" properties of the corresponding graphs. This is due, in part, to the fact that the graph of a nil subsemigroup of $T_{X}$ may be interpreted as a partial order on $X$. Graph closed nil subsemigroups are therefore the prime objects of our investigation. In particular, we determine the maximal nil subsemigroups of $T_{X}$ and the maximal nilpotent semigroups of given nilpotency index and graph type. For finite $X$, we compute the order of closed nil subsemigroups of given nilpotency index and graph type. Furthermore, still for finite $X$, the number of isomorphism classes of maximal nil subsemigroups is determined and we show that every graph closed subsemigroup of a maximal nil semigroup $N$ is a meta-ideal of $N$.

Throughout this paper $E(S)$ denotes the set of idempotents of $S, N$ denotes the natural numbers, the number of elements in a set $X$ is denoted by $|X|$ and mappings are composed from right to left. For a finite set $X$, say $X=\{1,2, \ldots, n\}$, we use the notation of $\left[1\right.$, p. 54] for elements of $T_{X}$, namely $\alpha=\left(i_{1} i_{2} \cdots i_{n}\right)$ is the function $\alpha(j)=i_{j}$ for all $j \in X$. This notation is particularly useful for com-

Received by the editors October 1, 1974.

AMS (MOS) subject classifications (1970). Primary 20M20; Secondary 05C20.

Key words and phrases. Transformation semigroup, nil semigroup, idealizer, partially ordered set, digraph, algebraic graph, idealized graph. 
puting products, since $\left(i_{1} i_{2} \cdots i_{n}\right)\left(j_{1} j_{2} \cdots j_{n}\right)=\left(i_{j_{1}} i_{j_{2}} \cdots i_{j_{n}}\right)$.

2. Algebraic graphs. Let $X$ be a set and $G$ a directed graph on $X$. For any $i, j \in X$, we shall write $i \rightarrow j \in G$ to mean that the directed edge $(i, j)$ belongs to the graph $G$. A directed graph $G$ is said to be transitive if for all $i, j$ and $k$ in $X$, $i \rightarrow j \in G$ and $j \rightarrow k \in G$ implies that $i \rightarrow k \in G$.

2.1. Definition. A transitive graph $G$ on a set $X$ is said to be algebraic on $X$ if for all $i \in X$ there exists $j \in X$ such that $i \rightarrow j \in G$.

Let $S_{X}$ denote the set of all subsemigroups of the full transformation semigroup $T_{X}$ for a set $X$, and let $G_{X}$ be the set of all algebraic graphs on $X$. We introduce a function $g$ from $S_{X}$ to $G_{X}$ and a function $S$ from $G_{X}$ to $S_{X}$ such that $g \cdot S$ is the identity on $G_{X}$ and $S \cdot g$ is a closure operator on $S_{X}$.

2.2. Definition. Let $S \in S_{X}$. Construct a directed graph $g(S)$ on $X$ as follows: for each $i, j \in X, i \rightarrow j \in g(S)$ iff there exists $\alpha \in S$ such that $\alpha(i)=j$.

It is clear that for each semigroup $S \in S_{X}, g(S) \in G_{X}$ and so we have a function $g: S_{X} \rightarrow G_{X}$.

2.3. Definition. Let $G \in G_{X}$. Define $S(G)=\left\{\alpha \in T_{X} \mid\right.$ for each $i \in X$, $\alpha(i)=j$ implies that $i \rightarrow j \in G\}$.

Since $G$ is algebraic, it follows that $S(G)$ is a semigroup, i.e. $S(G) \in S_{X}$.

The following lemma is an immediate consequence of the above definitions.

2.3. LEMma. (i) If $S_{1}, S_{2} \in S_{X}$ and $S_{1} \subset S_{2}$, then $g\left(S_{1}\right) \subset g\left(S_{2}\right)$.

(ii) If $G_{1}, G_{2} \in G_{X}$ and $G_{1} \subset G_{2}$, then $S\left(G_{1}\right) \subset S\left(G_{2}\right)$.

2.4. THEOREM. $g \cdot S$ is the identity on $G_{X}$ and $S \cdot g$ is a closure operator on $S_{X}$.

Proof. First we prove that $g \cdot S$ is the identity on $G_{X}$. Let $G \in G_{X}$ and suppose that $i \rightarrow j \in G$. Define $\alpha \in T_{X}$ by $\alpha(i)=j$, while for each $k \in$ $X \backslash\{i\}, \alpha(k)=l$ where $l$ is any element of $X$ such that $k \rightarrow l \in G$. At least one such $l$ must exist for each $k$, since $G$ is algebraic. Thus $\alpha \in S(G)$, and so $i \rightarrow j$ $\in g \cdot S(G)$. Thus $G \subset g \cdot S(G)$. Conversely, if $i \rightarrow j \in g \cdot S(G)$, there exists $\alpha \in S(G)$ such that $\alpha(i)=j$. This implies that $i \rightarrow j \in G$ and so $g \cdot S(G) \subset G$. Thus $G=g \cdot S(G)$.

It is easily seen that $S \cdot g$ is a closure operator on $S_{X}$, i.e. that $S \cdot g$ has the following properties: (i) for each $S \in S_{X}, S \subset S \cdot g(S)$, (ii) if $S, T \in$ $S_{X}$ and $S \subset T$, then $S \cdot g(S) \subset S \cdot g(T)$, and (iii) $S \cdot g$ is idempotent.

2.5. Definition. For each $S \in S_{X}$, let $\bar{S}=S \cdot g(S)$.

2.6. Definition. For each directed graph $G$ on a set $X$, we define a directed graph $I(G)$, called the idealized graph of $G$ (for reasons which will be 
apparent in Lemma 2.10), as follows: let $i, j \in X$. Then $i \rightarrow j \in I(G)$ iff for all $k \in X$ :

(i) $j \rightarrow k \in G$ implies that $i \rightarrow k \in G$, and

(ii) $k \rightarrow i \in G$ implies that $k \rightarrow j \in G$.

Observe that the idealized graph of any algebraic graph contains the directed edges $i \rightarrow i \forall i \in X$.

2.7. ThEOREM If $G \in G_{X}$, then $I(G) \in G_{X}$ and $G \subset I(G)$.

Proof. Suppose $G \in G_{X}$. To show that $I(G) \in G_{X}$, it is sufficient to show that $G \subset I(G)$ and $I(G)$ is transitive. Let $i \rightarrow j \in G$. Then for all $k \in X$, if $j \rightarrow k \in G$, then $i \rightarrow k \in G$, while if $k \rightarrow i \in G$, then $k \rightarrow j \in G$. Thus $i \rightarrow j \in I(G)$; and so $G \subset I(G)$. Now, to prove that $I(G)$ is transitive, let $i \rightarrow$ $j \in I(G), j \rightarrow k \in I(G)$. We must show that $i \rightarrow k \in I(G)$. Let $k \rightarrow l \in G$. Then $j \rightarrow l \in G$ and so $i \rightarrow l \in G$. On the other hand, if $l \rightarrow i \in G$, then $l \rightarrow$ $j \in G$ and thus $l \rightarrow k \in G$. Therefore $i \rightarrow k \in I(G)$.

Let us return now to a consideration of the closure operator on $S_{X}$. The natural course to take, when one has a closure operator, is to single out the "closed" objects for investigation.

2.8. Definition. A semigroup $S \in S_{X}$ is said to be graph closed if $S=\bar{S}$.

It is clear that not all elements of $S_{X}$ are graph closed. For example, the only subgroups of $T_{X}$ which are graph closed are the trivial groups. Thus graph closed semigroups are far removed from groups. An interesting class of graph closed semigroups, as we shall see, are the graph closed nil subsemigroups of $T_{X}$. We begin with an investigation of the properties of graph closed semigroups in general. The first observation to be made is that a graph closed semigroup is completely described by its graph. A convenient presentation of a graph closed semigroup on a finite set can be obtained by a table in the following way: let $X=$ $\{1,2, \ldots, n\}$, and let $S \in S_{X}$ be graph closed. Make a table with $n$ columns, one for each element of $X$, and in the column corresponding to $i \in X$, write down all elements $j \in X$ such that $i \rightarrow j \in g(S)$. Then an element $\alpha$ of $S$ is obtained by choosing one element from each column, say $j_{i}$ is chosen from the $i$ th column for each $i \in X$, and setting $\alpha=\left(j_{1} j_{2} \cdots j_{n}\right)$. Moreover, each element of $S$ is obtained in this way, and distinct choices obviously give different elements of $S$. For each $i \in X$, let $n_{i}$ be the number of elements in the $i$ th column, i.e. $n_{i}$ is the number of elements $j \in X$ such that there exists $\alpha \in S$ with $\alpha(i)=j$.

2.9. Theorem (Counting Lemma). Let $X$ be a finite set and $S \in S_{X}$ be graph closed. Then $|S|=\Pi_{i \in X} n_{i}$.

For example, let $X=\{1,2,3,4\}$ and $G$ be the algebraic graph 


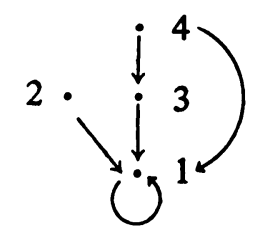

The table for $S(G)$ is

\begin{tabular}{|l|l|l|l|}
\hline 1 & 2 & 3 & 4 \\
\hline 1 & 1 & 1 & 1 \\
\hline
\end{tabular}

and $S(G)=\{(1111),(1113)\}$. In addition, consider the idealized graph $I(G)$ of $G$ :

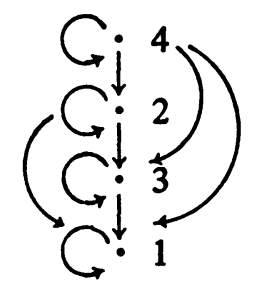

with table

\begin{tabular}{|l|l|l|l|}
\hline 1 & 2 & 3 & 4 \\
\hline 1 & 1 & 1 & 1 \\
\hline \multirow{2}{*}{2} & 2 & 3 & 2 \\
\cline { 2 - 4 } 3 & & 3 \\
\cline { 2 - 4 } & & \\
\cline { 3 - 4 }
\end{tabular}

The order of $S \cdot I(G)$ is (3) (2) (4) = 24. It is easily seen in this example that $S(G)$ is an ideal in $S \cdot I(G)$. In fact, $S \cdot I(G)$ is the idealizer of $S(G)$ and the following lemma asserts that this is true in general.

2.10. Lemma. Let $S \in S_{X}$ be graph closed. Then the idealizer $I(S)$ of $S$ in $T_{X}$ is graph closed and $g \cdot I(S)=I \cdot g(S)$.

Proof. We prove that $I(S)=S \cdot I \cdot g(S)$ whence $I(S)$ is graph closed and by (2.4), $g \bullet I(S)=g \cdot S \cdot I \cdot g(S)=I \cdot g(S)$. Let $G=I \cdot g(S)$. We begin by showing that $S(G) \subset I(S)$. Let $\alpha \in S(G)$ and $\beta \in S$. Then for each $i \in X, i \rightarrow \beta(i) \in g(S)$ and $\beta(i) \rightarrow \alpha \beta(i) \in G$. Thus $i \rightarrow \alpha \beta(i) \in g(S)$. 
Since $S$ is graph closed, $\alpha \beta \in S$. Similarly, $\beta \alpha \in S$. Thus $\alpha \in I(S)$ and so $S(G) \subset I(S)$.

Conversely we prove that $I(S) \subset S(G)$. Suppose this is not so, and choose $\beta \in I(S) \backslash S(G)$. Then there exist $i, j \in X$ such that $i \rightarrow j \notin G$ but $\beta(i)=j$. It follows that for some $k \in X$, either

(i) $k \rightarrow i \in g(S)$ but $k \rightarrow j \notin g(S)$, or else

(ii) $j \rightarrow k \in g(S)$ but $i \rightarrow k \notin g(S)$.

If (i) is the case, then there exists $\alpha \in S$ such that $\alpha(k)=i$. However, $\beta \alpha \in S$ and $\beta \alpha(k)=\beta(i)=j$ and so $k \rightarrow j \in g(S)$, a contradiction. If (ii) holds, then there exists $\alpha \in S$ such that $\alpha(j)=k$. But $\alpha \beta \in S$ and $\alpha \beta(i)=\alpha(j)=k$, whence $i \rightarrow k \in g(S)$, again a contradiction. Thus $I(S) \backslash S(G)=\varnothing$.

This lemma implies that for each graph closed semigroup $S_{0} \in S_{X}$, a sequence of graph closed semigroups $S_{i} \in S_{X}$ can be found such that $S_{0} \subset S_{1} \subset$ $\cdots$ and $S_{i-1} \Delta S_{i}$ for each $i \geqslant 0$. However, even for finite $X$, it is not possible in general to obtain such a chain with $S_{i}=T_{X}$ for some $i$, i.e. a graph closed subsemigroup of $T_{X}$ is not necessarily a meta-ideal of $T_{X}$. For this would be equivalent to the following: for all graph closed $S \in S_{X}, I \cdot g(S)=g(S)$ implies that $g(S)$ is the graph of $T_{X}$, namely the complete graph on $X$, and if $S$ is graph closed, then $S=T_{X}$. But consider the following example. Let $X=$ $\{1,2,3\}$ and $G$ be the algebraic graph on $X$ given by

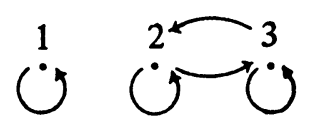

Clearly, $I(G)=G$ and $G$ is not the complete graph on $X$. In the last section, it will be shown that for finite $X$, any graph closed subsemigroup of a maximal nil semigroup in $T_{X}$ is a meta-ideal of the maximal nil semigroup.

The final observation of this section is the not unexpected result that two isomorphic graph closed semigroups are embedded in $T_{X}$ in the same way iff their graphs are isomorphic.

2.11. TheOREM. Let $S_{1}$ and $S_{2}$ be graph closed subsemigroups of $T_{X}$. Then $g\left(S_{1}\right) \simeq g\left(S_{2}\right)$ iff there exists an automorphism $h$ of $T_{X}$ such that $h$ induces an isomorphism between $S_{1}$ and $S_{2}$.

Proof. Let $g\left(S_{1}\right) \simeq g\left(S_{2}\right)$ be an isomorphism $\alpha$. Then $\alpha$ can be considered as an element of $\operatorname{Sym}(X) \subset T_{X}$. Let $c_{\alpha}: T_{X} \rightarrow T_{X}$ denote conjugation by $\alpha$, i.e. $c_{\alpha}(\gamma)=\alpha \gamma \alpha^{-1}$ for all $\gamma \in T_{X}$. Then $c_{\alpha} \in \operatorname{Aut}\left(T_{X}\right)$. We prove that $c_{\alpha} \mid S_{1}$ is an isomorphism from $S_{1}$ onto $S_{2}$. It is sufficient to prove that $c_{\alpha}\left(S_{1}\right) \subset S_{2}$ since by symmetry of argument, $c_{\alpha}^{-1}\left(S_{2}\right) \subset S_{1}$ and so $c_{\alpha}$ is surjective, whence it is bijective. Let $\gamma \in S_{1}$. Then $c_{\alpha}(\gamma)=\alpha \gamma \alpha^{-1}$. We must 
show that for all $i \in X, i \rightarrow \alpha \gamma \alpha^{-1}(i) \in g\left(S_{2}\right)$. Let $j=\alpha^{-1}(i)$, and $k=\gamma(j)$. Then $j \rightarrow k \in g\left(S_{1}\right)$ and so $\alpha(j) \rightarrow \alpha(k) \in g\left(S_{2}\right)$. But $\alpha(j)=i, \alpha(k)=$ $\alpha \gamma(j)=\alpha \gamma \alpha^{-1}(i)$ and so $i \rightarrow \alpha \gamma \alpha^{-1}(i) \in g\left(S_{2}\right)$. Thus $c_{\alpha} \mid S_{1}$ is an isomorphism from $S_{1}$ onto $S_{2}$.

Conversely, suppose there exists an automorphism $h$ of $T_{X}$ whose restriction to $S_{1}$ is an isomorphism from $S_{1}$ onto $S_{2}$. Since every automorphsim of $T_{X}$ is inner [2], there exists $\alpha \in \operatorname{Sym}(X)$ such that $h=c_{\alpha}$. Now $\alpha$ is a bijection on $X$. We prove that $\alpha$ preserves direction. Let $i \rightarrow j \in g\left(S_{1}\right)$. Then there exists $\beta \in$ $S_{1}$ such that $\beta(i)=j$. Let $c_{\alpha}(\beta)=\gamma \in S_{2}$. Then $\beta=\alpha^{-1} \gamma \alpha$ and so $\alpha^{-1} \gamma \alpha(i)$ $=j$, whence $\gamma(\alpha(i))=\alpha(j)$ and so $\alpha(i) \rightarrow \alpha(j) \in g\left(S_{2}\right)$. By symmetry, $\left(c_{\alpha}\right)^{-1}$ $=c_{\alpha}^{-1}$ also preserves direction. Thus $g\left(S_{1}\right) \simeq g\left(S_{2}\right)$.

3. Nil semigroups of $T_{X}$. In this section we show that the set of graph closed nil subsemigroups of $T_{X}$ is a very tractable class of graph closed elements of $S_{X}$ to study. The principal observation to be made is that the graph of a nil element of $S_{X}$ "is" a partial order on $X$ having certain properties. Many of the observations to be made about nil graph closed elements of $S_{X}$ are intuitively clear from a consideration of the graph of the semigroup.

3.1. Lemma. Let $S \in S_{X}$ be nil with zero $\xi$. Then the set of fixed points of each element of $S$ is $\xi(X)$.

Proof. Let $\alpha \in X$. For each $i \in \xi(X), i=\xi(j)$ for some $j \in X$ and so $\alpha(i)=\alpha \xi(j)=\xi(j)=i$. Thus $\alpha$ fixes $i$. Now suppose that for some $j \in X$, $\alpha(j)=j$. Then for each positive integer $n, \alpha^{n}(j)=j$. Since $S$ is nil, $\alpha^{n}=\xi$ for some $n$. Thus $\xi(j)=j$ and so $j \in \xi(X)$.

3.2. Definition. For a nil semigroup $S \in S_{X}$ with zero $\xi$, let $\operatorname{Fix}(S)=$ $\xi(X)$. In particular, $\operatorname{Fix}(\{\xi\})$ is denoted by $\operatorname{Fix}(\xi)$.

We now establish that the relation on $X$ determined by $g(S)$ for nil $S$ is a partial order with set of minimal elements $\operatorname{Fix}(S)$ and having the property that every element of $X \backslash \operatorname{Fix}(S)$ is greater than exactly one element of $\operatorname{Fix}(S)$.

3.3. Definition. Let $S \in S_{X}$ be nil. Then for $i, j \in X$, let $i \geqslant_{S} j$ iff $i=j$ or there exists $\alpha \in S$ such that $\alpha(i)=j$.

This is clearly a reflexive, transitive relation on $X$. Suppose that $i \geqslant_{s} j$ and $j \geqslant_{S} i$. If $i \neq j$, then there exist $\alpha, \beta \in S$ such that $\alpha(i)=j$ and $\beta(j)=i$ whence $\beta \alpha(i)=i$ and so $i \in \operatorname{Fix}(S)$. But then $i=j$, a contradiction. Thus $i=j$ and $\geqslant_{S}$ is antisymmetric, hence a partial order on $X$ said to be induced by $S$. We remark that if $S, T \in S_{X}$ are nil and $S \subset T$, then $i \leqslant_{S} j$ implies $i \leqslant_{T} j$.

3.4. LeMmA. The partial order on $X$ induced by a nil semigroup $S \subset T_{X}$ 
has minimal elements, and every element which is not minimal is greater than exactly one minimal element. The set of all minimal elements is $\operatorname{Fix}(S)$.

Proof. Let $i \in \operatorname{Fix}(S)$. Then $\alpha(i)=i$ for all $\alpha \in S$ and thus $i$ is minimal for $\geqslant_{S}$. Suppose conversely that $i \in X$ is minimal. Then $\xi(i)=i \in \operatorname{Fix}(S)$, where $\xi$ is the zero of $S$.

It is clear that for every $i \in X, \xi(i)$ is the unique element of $\operatorname{Fix}(S)$ which is less than or equal to $i$.

Let $X$ be a set. For the remainder of this section, $S$ shall denote a nil element of $S_{X}$ with zero $\xi$.

3.5. Definition. For each $i \in X$, let $X_{i}=X_{i}(S)=\left\{j \in X \mid j \geqslant_{S} i\right\}$.

The preceding lemma implies that the set $\left\{X_{i} \mid i \in \operatorname{Fix}(S)\right\}$ is a partition of $X$.

3.6. THEOREM. For each $i \in \operatorname{Fix}(S)$, there exists a nil semigroup $S_{i} \subset$ $T_{X_{i}}$ such that

(i) $\left|\operatorname{Fix}\left(S_{i}\right)\right|=1$,

(ii) each $S_{i}$ is a retract of $S$ in such a way that $S_{i} S_{j}=\{\xi\}$ for all $i, j \in$ $\operatorname{Fix}(S), i \neq j$,

(iii) $S$ is a subdirect product of the $S_{i}$. If $S$ is graph closed, then $S \simeq$ $\Pi_{i \in \mathrm{Fix}(S)} S_{i}$ and each $S_{i}$ is graph closed.

Proof. First, observe that (3.4) implies that for each $i \in \operatorname{Fix}(S)$ and each $\alpha \in S, \alpha \mid X_{i} \in T_{X_{i}}$. Let $S_{i}=\left\{\alpha\left|X_{i}\right| \alpha \in S\right\}$. It is obvious that $S_{i}$ is a nil subsemigroup of $T_{X_{i}}$ with zero $\xi \mid X_{i}$, and $\xi\left(X_{i}\right)=\{i\}$. Thus $\left|\operatorname{Fix}\left(S_{i}\right)\right|=1$. Define for each $i \in \operatorname{Fix}(S)$ a function $I_{i}: S_{i} \rightarrow S$ as follows: for $\alpha \in S_{i}$, let $I_{i}(\alpha) \mid X_{i}=$ $\alpha$ and $I_{i}(\alpha)\left|X \backslash X_{i}=\xi\right| X \backslash X_{i}$. It is clear that $I_{i}$ is an injective homomorphism. Now let $\Pi_{i}: S \rightarrow S_{i}$ be the restriction homomorphism. We have $\Pi_{i} \cdot I_{i}=\mathrm{id}_{S_{i}}$ and so $S_{i}$ is a retract of $S$. By the definition of $I_{i}$, (the images of) $S_{i}$ and $S_{j}$ satisfy $S_{i} S_{j}=\{\xi\}$ for $i, j \in \operatorname{Fix}(S), i \neq j$. From the definition of the $S_{i}$, statement (iii) is clear.

REMARK. If $\left|X_{i}\right| \leqslant 2$ for some $i \in \operatorname{Fix}(S)$, then $\left|S_{i}\right|=1$. that

3.7. Definition. Let $G \in G_{X}$. If there exists a subset $F(G)$ of $X$ such

(i) for all $i \in X, i \rightarrow i \in G$ iff $i \in F(G)$, and

(ii) for all $j \in X$, there exists a unique $i \in F(G)$ such that $j \rightarrow i \in G$, then $G$ is said to be a nil graph. For each $i \in X$, let $i_{F}$ be the unique element of $F(G)$ whose existence is asserted by (ii).

It is readily apparent that if $S \in S_{X}$ is nil, then $g(S)$ is nil, while if $G \in$ $G_{X}$ is nil, then $S(G)$ is nil. We denote by $\leqslant_{G}$ the partial order on $X$ induced by 
$S(G)$. For $i, j \in X$ with $i \leqslant_{G} j$, the closed interval with end points $i$ and $j$ shall be denoted by $[i, j]_{G}$. If $[i, j]_{G}$ is a chain with $n+1$ elements, it is said to be of length $n$. Finally, a nil graph for which every chain is of length less than or equal to $k$, and some chain is of length $k$, is called a $k$-nilpotent graph. For a nilpotent graph $G$ on a set $X$, we can define a function from $X$ into $N$, called the height function on $X$ corresponding to $G$.

3.8. Definition. Let $G \in G_{X}$ be $k$-nilpotent and consider the partial order $\leqslant_{G}$ induced on $X$ by $G$. The function $h_{G}: X \rightarrow\{0,1,2, \ldots, k\}$ defined by setting $h_{G}(i)$ equal to the length of the longest chain in $\left[i_{F}, i\right]_{G}$ is called the height function on $X$ induced by $G$.

A $k$-nilpotent semigroup is a nilpotent semigroup of nilpotency index $k$. If $S \in S_{X}$ is $k$-nilpotent, then $g(S)$ is $k$-nilpotent. For nilpotent $S$, let $h_{S}=h_{g(S)}$.

3.9. Lemma. (i) $h_{G}$ is surjective.

(ii) $h_{G}(i)=0$ iff $i \in F(G)$.

(iii) If $i>_{G} j$, then $h_{G}(i)>h_{G}(j)$. Thus $h_{G}$ preserves strict inequality.

Let $n$ be a nonnegative integer. We shall refer to any surjective function $h: X \rightarrow\{0,1,2, \ldots, n\}$ as a height function on $X$. If $\left|h^{-1}(0)\right|=1$, then $h$ is said to be a proper height function.

As an example of how the height function aids our geometric insight, we give a short proof of the well-known result that a finite nil semigroup is nilpotent.

THEOREM. Let $S$ be a finite nil semigroup, $|S|=n$. Then $S^{n}=\{0\}$.

Proof. $S$ can be considered as a nil subsemigroup (via left translations) of $T_{X}$ where $X=S^{1}$. Then $\operatorname{Fix}(S)=\{0\}$, and $h_{S}(x) \leqslant n-1$ for any $x \in S$, since $|S|=n$. Thus for any $x_{1}, x_{2}, \ldots, x_{n} \in S$,

$$
h_{S}\left(x_{n}\right)>h_{S}\left(x_{n-1} x_{n}\right)>h_{S}\left(x_{n-2} x_{n-1} x_{n}\right)>\cdots>h_{S}\left(x_{1} x_{2} \cdots x_{n}\right)
$$

by (3.9)(iii) and so $h_{S}\left(x_{1} x_{2} \cdots x_{n}\right)=0$ whence $x_{1} x_{2} \cdots x_{n} \in \operatorname{Fix}(S)=\{0\}$.

COROLlaRY. Let $S$ be a finite nil semigroup, $|S|=n$. The index of nilpotency is $n$ iff $S$ is cyclic.

Proof. Clearly, if $S$ is cyclic, the index of nilpotency of $S$ is $n$. Conversely, if $S^{n-1} \neq\{0\}$, there exist $x_{1}, x_{2}, \ldots, x_{n-1} \in S$ such that $x_{1} x_{2} \ldots$ $x_{n-1} \neq 0$.

Thus $x_{2} \neq 0, x_{2} x_{3} \neq 0, \ldots, x_{2} x_{3} \cdots x_{n-1} \neq 0$ and no two are equal. Since $x_{1}, x_{1} x_{2}, \ldots, x_{1} x_{2} \cdots x_{n-1}$ are also nonzero and no two are equal, left translation by $x_{1}$ on $S^{1}$ has rank $n$. But the graph of $S$ is a chain, namely the transitive closure of 


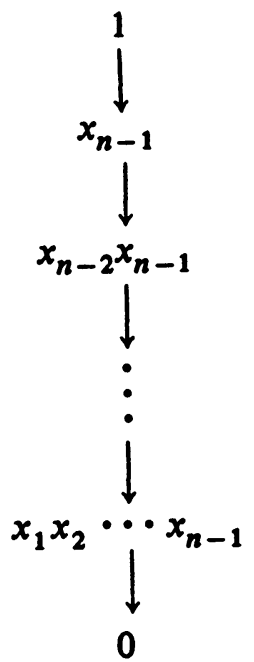

and thus $x_{1}^{n-1} \neq 0$, whence $S=\left\{x_{1}, x_{1}^{2}, \ldots, x_{1}^{n-1}, 0\right\}$.

4. Maximal nil semigroups in $T_{X}$. We concern ourselves now with the determination of the maximal nil semigroups, and more generally, the maximal $k$ nilpotent semigroups in $T_{X}$ for any set $X$. It is sufficient by (3.4) to study nil semigroups $S$ for which $\mid$ Fix $(S) \mid=1$, since maximal nilpotent (and hence maximal nil) semigroups are readily seen to be graph closed.

4.1. TheOREM. Let $\xi \in E\left(T_{X}\right)$ be such that $\mid$ Fix $(\xi) \mid=1$. Then a nil semigroup $N$ containing $\xi$ is maximal nil iff $N$ is graph closed and $g(N)$ is a chain with minimum element $\xi(X)$.

Proof. Let $\xi$ be the zero of $N$ and suppose that $\xi(X)=i$. Then the graph of $\{\xi\}$ is

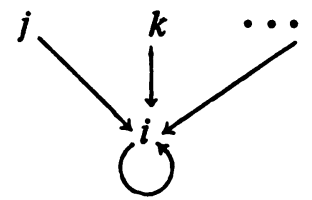

where $X=\{i, j, k, \ldots\}$. This graph must be a subgraph of the graph of any nil subsemigroup of $T_{X}$ containing $\xi$. A maximal such graph is a chain with least element $i$ and the graph closed semigroup corresponding to such a chain is clearly a maximal nil subsemigroup of $T_{X}$.

4.2. Corollary. Let $X$ be a finite set and $\xi \in E\left(T_{X}\right),|F i x(\xi)|=1$. Then any maximal nil subsemigroup of $T_{X}$ with $\xi$ for zero has order $(|X|-1)$ !.

ProOF. This is immediate by the counting lemma. 
It now follows from (3.4) that if $\xi \in E\left(T_{X}\right)$, any maximal nil subsemigroup in $T_{X}$ having $\xi$ as zero is graph closed, and its graph is a disjoint union of chains, each with minimum element.

4.3. Corollary. Let $\xi \in E\left(T_{X}\right)$, for $X$ finite. Then if $n_{i}=\left|X_{i}\right|$ for each $i \in \operatorname{Fix}(\xi)$, the order of any maximal nil subsemigroup of $T_{X}$ having $\xi$ as zero is $\Pi_{i \in \operatorname{Fix}(\xi)}\left(n_{i}-1\right) !$

It would certainly appear that graph closed semigroups are described completely by their graphs and one might naturally expect to be able to describe the isomorphism classes of graph closed nil semigroups in terms of graphs. By (2.11), if $G$ is a nil graph on $X$, the set of all graph closed nil subsemigroups of $T_{X}$ whose graphs are isomorphic to $G$ is contained in an isomorphism class. In general, this containment is proper, i.e. there will exist graph closed nil semigroups which are isomorphic to $S(G)$, but whose graphs are not isomorphic to $G$. Such semigroups must of course be embedded in $T_{X}$ differently, i.e. they are not isomorphic via an automorphism of $T_{X}$.

The next result describes the isomorphism classes of maximal nil subsemigroups of $T_{X}$ for a finite set $X$.

For $n \in N$, let $\pi(n)$ denote the set of all unordered partitions of $n$. For fixed $k \in N, k \leqslant n$, define an equivalence relation $\stackrel{k}{\sim}$ on $\pi(n)$ as follows: let $p \in \pi(n)$ and let $r_{k}(p)=\{m \in p \mid m>k\}$. Then for $p, q \in \pi(n)$, put $p \stackrel{k}{\sim} q$ iff $r_{k}(p)=r_{k}(q)$.

Now, each nil semigroup $N \in S_{X}$ determines a partition of $|X|$, namely $p(N)=\left\{\left|X_{i}\right| \mid i \in \operatorname{Fix}(N)\right\}$.

4.4. TheOREM. Let $N, M$ be maximal nil subsemigroups of $T_{X}$. Then $N \simeq M$ iff $p(N) \stackrel{2}{\sim} p(M)$.

Proof. Suppose that $N \simeq M$. Without loss of generality, let $\operatorname{Fix}(N)=$ $\{1,2, \ldots, r\}, \operatorname{Fix}(M)=\{1,2, \ldots, s\}$, and $p(N)=\left\{n_{1}, n_{2}, \ldots, n_{r}\right\}, p(M)=$ $\left\{m_{1}, m_{2}, \ldots, m_{s}\right\}$. We assume that the labelling is such that $n_{1} \geqslant n_{2} \geqslant \cdots \geqslant$ $n_{r}$ and $m_{1} \geqslant m_{2} \geqslant \cdots \geqslant m_{s}$. If $n_{1}>m_{1}$ then either $n_{1}=2$ or $n_{1}>2$. If $n_{1}=2$, then $p(N) \stackrel{2}{\sim} p(M)$ and we are done. Suppose $n_{1}>2$. Then $N$ contains an element nilpotent of index $n_{1}-1$, while $M$ has no elements of nilpotency index greater than $m_{1}-1$. This is a contradiction, since $N \simeq M$. Thus $n_{1}=m_{1}$. Let $k$ be the greatest integer such that $n_{i}=m_{i}$ for all $i \leqslant k$. Now if $k=\min \{r, s\}$, then $r=s$ and $p(N)=p(M)$. Suppose that $k<\min \{r, s\}$. Let $N \simeq \prod_{i=1}^{r} N_{i}, M \simeq \prod_{i=1}^{s} M_{i}$ as described in (3.6). Now put

$$
A=\prod_{i=1}^{k} N_{i}, \quad B=\prod_{i=k+1}^{r} N_{i}, \quad C=\prod_{i=1}^{k} M_{i}, \quad \text { and } \quad D=\prod_{i=k+1}^{s} M_{i}
$$


We shall make use of the following counting functions: for any finite nil semigroup $S$ and nonnegative integer $n$, let $\eta_{S}(n)$ be the number of elements in $S$ of nilpotency index $n$, and $\mu_{S}(n)$ be the number of elements in $S$ of nilpotency index less than $n$. Now, suppose that $n_{k+1}<m_{k+1}$, and let $m=m_{k+1}-1$. If $m \leqslant 1$, then $p(N) \stackrel{2}{\sim} p(M)$. Suppose that $m>1$. Then

while

$$
\eta_{M}(m)=\eta_{C}(m) \cdot|D|+\mu_{C}(m) \cdot \eta_{D}(m)
$$

$$
\eta_{N}(m)=\eta_{A}(m) \cdot|B|+\mu_{A}(m) \cdot \eta_{B}(m)
$$

But $\eta_{B}(m)=0$, and by (2.11), $N_{i} \simeq M_{i}$ for all $i \leqslant k$, whence $A \simeq C$ and so (i) $\eta_{A}(m)=\eta_{C}(m)$, and (ii) $|A|=|C|$ which implies that $|B|=|D|$. Since $\mu_{C}(m) \eta_{D}(m)>0$, we have $\eta_{M}(m)>\eta_{N}(m)$, a contradiction to the fact that $N \simeq$ $M$. Thus $p(N) \stackrel{2}{\sim} p(M)$.

Conversely, suppose that $N$ and $M$ are maximal nil subsemigroups of $T_{X}$ such that $p(N) \underset{\sim}{\sim} p(M)$. As before, suppose that $p(N)=\left\{n_{1}, n_{2}, \ldots, n_{r}\right\}$, $p(M)=\left\{m_{1}, m_{2}, \ldots, m_{s}\right\}$ with $n_{1} \geqslant n_{2} \geqslant \cdots \geqslant n_{r}, m_{1} \geqslant m_{2} \geqslant \cdots \geqslant m_{s}$ and $N \simeq \Pi_{i=1}^{r} N_{i}, M \simeq \Pi_{i=1}^{s} M_{i}$. Now $n_{1} \leqslant 2$ iff $m_{1} \leqslant 2$ and if so, both $N$ and $M$ are singletons. Suppose then that $n_{1}, m_{1}>2$. Let $k$ be the greatest integer such that $n_{i}=m_{i}$ for all $i \leqslant k$. Then by (2.11), there is an isomorphism $\theta_{i}: N_{i} \rightarrow M_{i}$ for $i=1,2, \ldots, k$. Let $A=\Pi_{i=1}^{k} N_{i}, C=\Pi_{i=1}^{k} M_{i}$. The product map $\theta=\Pi_{i=1}^{k} \theta_{i}$ is an isomorphism of $A$ onto $C$ and since $N \simeq A, M \simeq C$ (the remaining factors in both product decompositions are zero), we have $N \simeq M$.

4.5. COROLLARY. There are $|\pi(|X|) / \stackrel{2}{\sim}|$ isomorphism classes of maximal nil subsemigroups of $T_{X}$.

We remark that if the only isomorphisms allowed are those induced by automorphisms of $T_{X}$, then there are $|\pi(|X|)|$ isomorphism classes.

Consider now the nilpotent subsemigroups of $T_{X}$. We shall describe the maximal nilpotent semigroups of given nilpotency index in terms of height functions on $X$.

4.6. Definition. Let $h: X \rightarrow\{0,1,2, \ldots, n\}$ be a proper height function on $X$. Denote by $G(h)$ the directed graph on $X$ defined as follows: for $i, j \in X, i \rightarrow j \in G(h)$ iff (i) $h(i)>h(j)$; or (ii) $i=j$ and $h(i)=0$.

4.7. Lemma. $G(h)$ is a nil graph with $F(G)=h^{-1}(0)$ and $h=h_{G(h)}$.

4.8. TheOREM. Let $h: X \rightarrow\{0,1,2, \ldots, k\}$ be a proper height function. Then there exists a maximum $k$-nilpotent subsemigroup $N=N(h)$ of $T_{X}$ such that $h_{N}=h$. Furthermore, $N$ is graph closed and $g(N)=G(h)$.

Proof. Let $N=S(G(h))$. Then $g(N)=G(h), N$ is graph closed and $k$ - 
nilpotent. Clearly $h_{N}=h$. Let $M \subset T_{X}$ be any nil semigroup such that $h_{M}=h$. We prove that $M \subset N$. Let $\gamma \in M$. Then for each $i \in X$, put $j=\gamma(i)$. Now $i \in \operatorname{Fix}(N)$ iff $h_{N}(i)=0=h(i)=h_{M}(i)$ iff $i \in \operatorname{Fix}(M)$, whence $j=i$. Otherwise, $i \neq j$ and so $h_{M}(i)>h_{M}(j)$, whence $h(i)>h(j)$ and so $i \rightarrow j \in g(N)$. Thus $\gamma \in N$ and we have $M \subset N$.

4.9. Corollary. For finite $X$, the order of $N(h)$ is computed as follows: let $n_{i}$ denote the number of elements of $X$ of height less than $i$, and let $m_{i}$ be the number of elements of height $i$ for $i=1,2, \ldots, n$. Then $|N(h)|=$ $\Pi_{k=1}^{n}\left(\sum_{i=1}^{k} n_{i}\right)^{m_{k}}$.

4.10. Corollary. Let $\xi \in E\left(T_{X}\right)$ be such that $|\xi(X)|=1$. Then a nil semigroup $N \subset T(X)$ with zero $\xi$ is a maximal $k$-nilpotent semigroup iff $\max \left\{h_{N}(i) \mid i \in X\right\}=k, N$ is graph closed, and $h_{N}(i)>h_{N}(j)$ implies that $i \rightarrow$ $j \in g(N)$ for all $i, j \in X$.

4.11. Corollary. Let $\left\{X_{\alpha} \mid \alpha \in \Lambda\right\}$ be a partition of $X$ and let $h_{\alpha}$ : $X_{\alpha} \rightarrow\left\{0,1,2, \ldots, m_{\alpha}\right\}$ be a proper height function for each $\alpha \in \Lambda$. If $\max \left\{m_{\alpha} \mid \alpha \in \Lambda\right\}$ exists, say it is $k$, then $h=\bigcup_{\alpha \in \Lambda} h_{\alpha}: X \rightarrow\{0,1,2, \ldots, k\}$ is a height function on $X$ and there exists a maximum $k$-nilpotent semigroup $N \subset T_{X}$ such that $h_{N}=h$.

4.12. Corollary. Let $N \in S_{X}$ be k-nilpotent. Then there exists a maximum $k$-nilpotent subsemigroup $M$ of $T_{X}$ such that $N \subset M$ and $h_{M}=h_{N}$.

In particular, for finite $X$, each nil $N \in S_{X}$ is contained in a maximum nil $M \in S_{X}$ such that $h_{N}=h_{M}$.

5. Ideal chains of nil semigroups.

5.1. THEOREM. Let $N, M \in S_{X}$ be maximal nilpotent of index $n$ and $m$ respectively. If $N \subset M$ then $N \Delta M$.

Proof. Let $\alpha \in N, \beta \in M \backslash N$. We must show that $\alpha \beta$ and $\beta \alpha$ belong to $N$. Since $N$ is maximal $n$-nilpotent, this will be done if we show that for all $i \in$ $X \backslash$ Fix $(N)$, (i) $h_{N}(i)>h_{N} \cdot \alpha \beta(i)$, and (ii) $h_{N}(i)>h_{N} \cdot \beta \alpha(i)$. The proofs of (i) and (ii) are similar, and we just prove (i). Let $i \in X \backslash$ Fix $(N)$. Note that $\operatorname{Fix}(M)=\operatorname{Fix}(N)$. Put $k=\beta(i), j=\alpha(k)$. We want to show that $h_{N}(i)>$ $h_{N}(j)$. If $h_{N}(i)<h_{N}(k)$, then there exists $\gamma \in N$ such that $\gamma(k)=i$. But then $\gamma \in M$ and so $\gamma \beta(i)=i$, whence $i \in \operatorname{Fix}(M)$, a contradiction. Thus $h_{N}(i) \geqslant$ $h_{N}(k)$. If $k \in \operatorname{Fix}(N)$, then $k=j$ and $h_{N}(i)>0=h_{N}(j)$. If $k \notin \operatorname{Fix}(N)$, then $h_{N}(k)>h_{N}(j)$ and so $h_{N}(i)>h_{N}(j)$.

For finite $X$, every graph closed subsemigroup of any maximal nil subsemigroup $N$ of $T_{X}$ is a meta-ideal of $N$. We need some preliminary results to estab- 
lish this, beginning with a result for arbitrary $X$.

5.2. Lemma. Let $N \in S_{X}$ be nil and let $i, j \in \operatorname{Fix}(N), i \neq j$. Then for any $x \in X_{i}, y \in X_{j}, x \rightarrow y \notin I \cdot g(N)$.

Proof. Suppose that for some $x \in X_{i}, y \in X_{j}, x \rightarrow y \in I \cdot g(N)$. Then since $y \rightarrow j \in g(N)$, we must have $x \rightarrow j \in g(N)$, and so $x \in X_{j}$, a contradiction.

Let $N_{i}, i \in \operatorname{Fix}(N)$, be the subsemigroup of $N$ as described in (2.11).

5.3. Corollary. $I \cdot g(N)=\bigcup_{i \in \operatorname{Fix}(N)} I \cdot g\left(N_{i}\right)$.

5.4. Theorem. Let $N \in S_{X}, X$ finite, be maximal nil and let $S$ be a graph closed subsemigroup of $N$. If $I \cdot g(S) \cap g(S) \cap g(S)$, then $S=N$.

Proof. By (5.3), we may assume that $\operatorname{Fix}(S)=\operatorname{Fix}(N)$ is a singleton. Suppose now that $S \neq N$, whence there exists an integer $n$ for which $\left|h_{S}^{-1}(n)\right|>$ 1. Let $m$ be the smallest such integer. Then $m>0$ by assumption, and the elements of $X$ of height less than $m$ form a chain under $\leqslant_{s}$, with a maximum element, say $k$. Let $i, j \in h_{S}^{-1}(m)$, whence $i \rightarrow k$ and $j \rightarrow k$ belong to $g(S)$. Now exactly one of $i \rightarrow j$ or $j \rightarrow i$ belongs to $g(N)$. Suppose that $i \rightarrow j$ belongs to $g(N)$. We show that $i \rightarrow j \in g(S)$ and hence $h_{S}(i)>h_{S}(j)$, a contradiction. Note that since $i \rightarrow j \in g(N), j \rightarrow i \notin g(S)$. Now let $l$ be any element of $X_{i}(S)$ of maximum height. Since $j \rightarrow l \in g(N)$ implies $j \rightarrow i \in g(N)$, we have $j \rightarrow l \notin g(N)$. Thus $l \rightarrow j \in g(N)$ and since $l$ is maximal in $X_{i}(S)$ under $\leqslant_{S}, l \rightarrow j \in I \cdot g(S)$. Thus $l \rightarrow j \in g(S)$. Now repeat this procedure for all elements of $X_{i}(S)$ of height one less than the maximum height in $X_{i}(S)$. We obtain that for all elements $l$ in $X_{i}(S)$ at this height, $l \rightarrow j \in g(S)$. By induction, we obtain $i \rightarrow j \in g(S)$, the contradiction we are seeking. Thus no two elements of $X$ have the same height relative to $S$ and so $S$ is maximal nil, whence $S=N$.

5.5. Corollary. Let $X$ be finite. Every graph closed subsemigroup of a maximal nil subsemigroup $N$ of $T_{X}$ is a meta-ideal of $N$.

\section{BIBLIOGRAPHY}

1. A. H. Clifford and G. B. Preston, The algebraic theory of semigroups. Vol. I, Math. Surveys, no. 7, Amer. Math. Soc., Providence, R.I., 1961. MR 24 \#A2627.

2. M. Petrich, The translational hull in semigroups and rings, Semigroup Forum 1 (1970), no. 4, 283-360. MR $42 \# 1919$.

DEPARTMENT OF MATHEMATICS, UNIVERSITY OF WESTERN ONTARIO, LONDON, ONTARIO, CANADA N6A 3K7 